European Association for the

Development of Renewable Energies,

Environment and Power Quality
International Conference on Renewable Energies and Power Quality (ICREPQ'09)

Valencia (Spain), 15th to 17th April, 2009

\title{
Tools for Assessing the Robustness Variation of Power System against Voltage Dips
}

\author{
P. Caramia ${ }^{2}$, C. Di Perna ${ }^{1}$, P.Varilone ${ }^{1}$, P. Verde ${ }^{1}$ \\ ${ }^{1}$ Dipartimento di Ingegneria Industriale \\ Università di Cassino \\ Via G. Di Biasio 43, 03043 Cassino (FR) Italia
}

Phone number:0039 0776 2993638, e-mail: verde@unicas.it,

\author{
${ }^{2}$ Dipartimenti per le Tecnologie \\ Università di Napoli Phartenope \\ Centro Direzionale Isola C4, 80100 Napoli \\ Phone number: 0039081 5476739,pierluigi.caramia@uniparthenope.it
}

\begin{abstract}
Electrical power system robustness is defined as the intrinsic capacity of an electrical power system to maintain assigned disturbance levels when external conditions change. In this paper the concept of robustness against is applied to the voltage dips, one of the most severe disturbances in power quality. Using graphical color schemes as immediate tools, improvements and/or degradations of the electrical power system robustness are assessed when structure modifications are implemented. The proposed tools are applied to real distribution systems.
\end{abstract}

\section{Key words}

Power quality, power system reliability, power transmission, power distribution, voltage dips.

\section{Introduction}

Voltage dip can originate in systems operating both in normal and in faulted conditions. This paper deals with voltage dips due to faults; the amplitude of voltage dip is here considered as the minimum RMS fault voltage.

The availability of tools permitting analysis and prediction of system performance in terms of voltage dips is of great interest. The voltage-dip frequency is, in general, so low that estimation to an acceptable degree of accuracy can be deduced only after several years of measurements.

Thus, theoretical analysis of electrical power systems aimed to forecast their performance in terms of voltage dips is important.

Voltage dips can be simulated using the Fault Position method (FP) [1]. This method can offer a global vision of the electrical power system response to faults, both for meshed systems and for radial networks.

The application of FP has been simplified by the introduction of the so called 'During Fault Voltage' (DFV) matrix, which is a bidimensional vector of voltages. Each element $(i, j)$ represents the vector of the during fault voltage in node $i$ when a short circuit occurs at node $\mathrm{j}$.
The DFV matrix illustrates the properties of all dips in all nodes of the system and aggregates information on dip propagation around the network.

Applying the FP method it is possible to have information regarding:

- propagation of voltage dips around the network;

- amplitude of voltage dips for all nodes;

- amplitude of voltage dips caused by each node;

- nodes where the faults are critical due to the voltage dips that they cause at other nodes;

- nodes where loads could experience the largest number of voltage dips.

To obtain immediate information on voltage dip performance for the entire system obtained by the FP method, a graphical color scheme has been proposed [2, 3]. The graphic visualization of the DFV matrix allows immediately ascertaining if (and where) external condition changes affect the electrical power system's ability to maintain dip severity at assigned levels. Fig.1 show the graphic visualization of the DFV matrix of a distribution system.

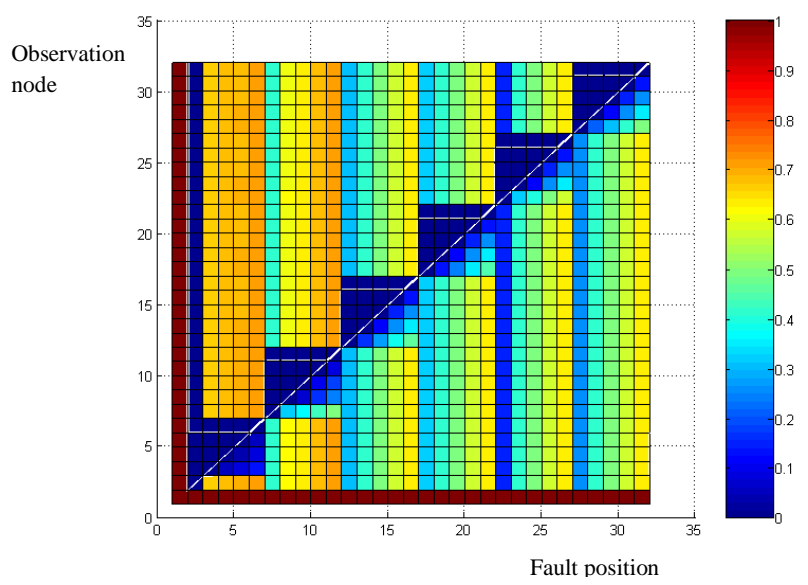

Fig. 1. Graphic visualization of a DFV matrix 
With reference to Fig.1, the DFV cells are represented by colors corresponding to their values by means of the grade scale reported in the same figure (blue colour for deep dip and red colour for no dip). With the exception of node 1 where a fault was never placed, each $\mathrm{j}^{\text {th }}$ column contains the during fault voltages for fault at node $j$ (affected area of node $\mathrm{j}$ ), each $\mathrm{i}^{\text {th }}$ row contains the during fault voltages at node $\mathrm{i}$ for faults in any other nodes (exposed area of node i).

It is clear that the node 2 presents the largest affected area (see the $2^{\text {nd }}$ column completely blue) and the smallest exposed area (see $2^{\text {nd }}$ row containing only few blue cells).

In others papers the concept of the power system robustness is introduced $[4,5]$. In particular, in [5] the analytical relationships between DFV variations and the short circuit power variations, through the terms of short circuit impedance matrix, were introduced.

Using the graphic color visualization, previously recalled, this paper aims to experimentally verify the relationships presented in [5]. The numerical cases refer to a real distribution networks..

\section{Robustness of Electrical Power Systems Against Voltage Dips: Definition And Linkage With DFV Matrix}

We defined electrical power system robustness against a disturbance as the intrinsic capacity of an electrical power system to maintain assigned disturbance levels when external conditions change [5].

Intrinsic capacity is the ability of the electrical power system to contain the voltage dip severity linked to the structural characteristics and to the features of the installed components. This definition emphasizes hardware characteristics such network configuration, installed generator powers, and the size and type of lines.

Disturbance levels are measured by several characteristics, including frequency of occurrence, duration, amplitude, number of phases, and phase angle jump.

External conditions play a significant role in the electrical power system response to a short circuit in the network. Relevant external conditions include pre-fault operations, network configuration, and the type and size of the distribution lines.

We must also ascertain parameters for immediate verification of system robustness. Short circuit power at a generic bus $\mathrm{k} S_{S C}^{k}$ represents an attractive global measure of the electrical power system strength.

If we compare every pair of nodes, we see that the larger the short circuit level of a node, the less severe the voltage dips. However, the larger short circuit level of a node, the more severe voltage dips that the node causes.

These two linkages can be viewed as the positive effect of the short circuit power on the voltage dips that the node experiences and as the negative effect of the short circuit power on the voltage dips that the node causes.

Such properties can be easily ascertained using the graphic visualization of the DFV matrices together with information on short circuit power levels of the nodes.
We can also examine the short circuit reactance matrix before and after a change in the electrical power system that affects the short circuit power of a node.

Assume that $X^{\text {new }}(k, k)$ is the short circuit reactance of the node $k$ after the change, and $V^{n e w}(f, k)$ is the during fault voltage of a generic node $f$ for a fault in the node $k$ after the change. Increasing the short circuit power of the generic node $k$ can produce dips in other nodes that are more or less severe than the dips that the same node causes before increasing its short circuit power. We compare the during fault voltage in generic node $f$ due to the fault in $k$ before increasing the short circuit power of node $k(V(f, k))$ with the same quantity after the change $\left(V^{\text {new }}(f, k)\right)$. For the diagonally dominant property of both short circuit reactance matrices before the change $\left[X_{S C}\right]$ and after the change $\left[X_{S C}^{\text {new }}\right]$, the conditions are:

$$
\begin{aligned}
& X(f, k) \leq X(k, k) ; \\
& X^{\text {new }}(f, k) \leq X^{\text {new }}(k, k) .
\end{aligned}
$$

Moreover, increasing the short circuit power of the node $k$ determines that

$$
X(k, k)>X^{\text {new }}(k, k) .
$$

Taking into account the above conditions, we have that $V(f, k) \geq V^{\text {new }}(f, k)$ if:

$$
1-\frac{X(f, k)}{X(k, k)} \geq 1-\frac{X^{\text {new }}(f, k)}{X^{\text {new }}(k, k)},
$$

this inequality can be developed as:

$$
\begin{aligned}
& X(f, k) \leq X^{\text {new }}(f, k) \frac{X(k, k)}{X^{\text {new }}(k, k)}, \\
& \text { with } \frac{X(k, k)}{X^{\text {new }}(k, k)}>1 .
\end{aligned}
$$

Relationship (4) can be expressed in terms of short circuit power, as follows:

$$
\begin{aligned}
& X(f, k) \leq X^{\text {new }}(f, k) \frac{S_{S C}^{k \text { new }}}{S_{S C}^{k}} \\
& \text { with } \frac{S_{S C}^{k \text { new }}}{S_{S C}^{k}}>1 ;
\end{aligned}
$$

where $S_{S C}^{k}$ and $S_{S C}^{k}{ }^{n e w}$ are respectively the short circuit powers at bus $k$ before and after the change in the power system.

If condition (5) is valid, then increasing the short circuit power in a node reflects negatively on the performance of other nodes in which $V^{\text {new }}(f, k) \leq V(f, k)$.

Varying the short circuit power of a node results in during fault voltages in other nodes depending on condition (5).

\section{Analysis of the Robustness of a Real Electrical Distribution System}

Robustness has been studied on the real electrical distribution power systems in South Italy. The simplified 


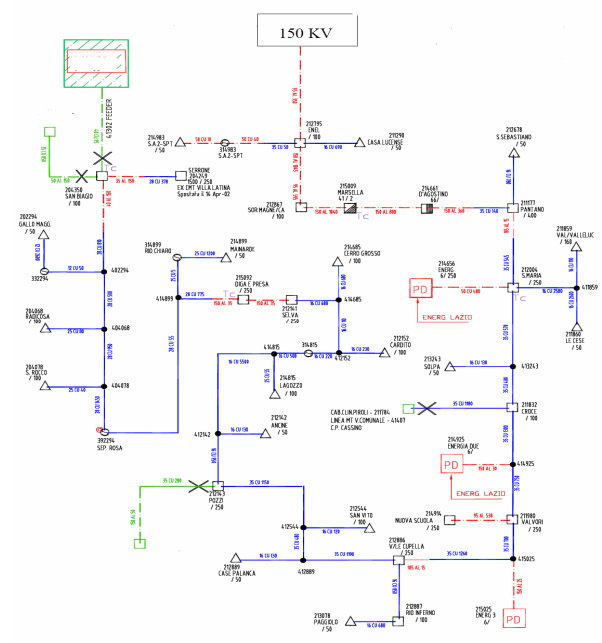

Fig. 2. Simplified scheme of the distribution system

scheme of the system is reported in Fig. 2. The studied distribution network represents a portion of a real $20 \mathrm{kV}$ distribution system supplying a mixture of industrial, commercial, and residential loads. The system consists of 36 distribution substations, $37153 \mathrm{Km}$ overhead lines and $4905 \mathrm{Km}$ underground cables..

The robustness is analyzed for different network configurations; the following cases are presented:

- case A: it is coincident with the real structure of the network;

- case B: it is obtained substituting some overhead lines (from L31 to L45) with cable lines;

- case C: it is obtained quadrupling the line L23 among the nodes 34 and 39 .

In each case, three phase solid faults are assumed.

In Figs. 3-5 the graphical representation of the DFV matrices for each case is reported.

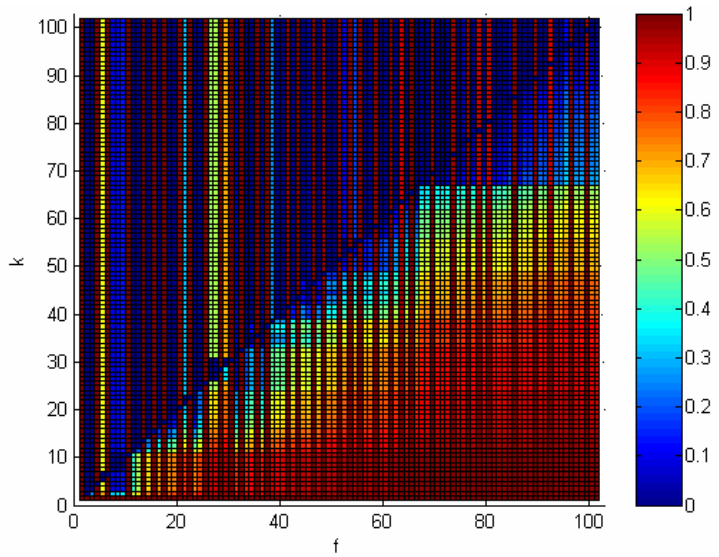

Fig. 3. Graphical visualization of the DFV matrix for case A

The graphical visualization of the DFV matrices achieve to obtain immediate information from the results of the FP method, but it remains rather difficult to compare each other the DFV matrices. To overcome such a difficulty, two new matrices, namely $\Delta X$ and $\Delta V$ matrix, are introduced. The terms $(f, k)$ of the matrices are, respectively:

$\Delta X(f, k)=X^{n e w}(f, k)-X(f, k)$,

$$
\Delta V(f, k)=V^{\text {new }}(f, k)-V(f, k) .
$$

The graphical representation with color grade of these new matrices allows better comparing, for example, case $B$ with case A.

To emphasize the variations a bicolor representation is used (Figs. 6 and 7). In each figure the blue color indicates a negative value while the red color indicates a positive value of the differences (6) and (7).

Fig.6 and Fig.7 can support the following analysis together with the Table I.

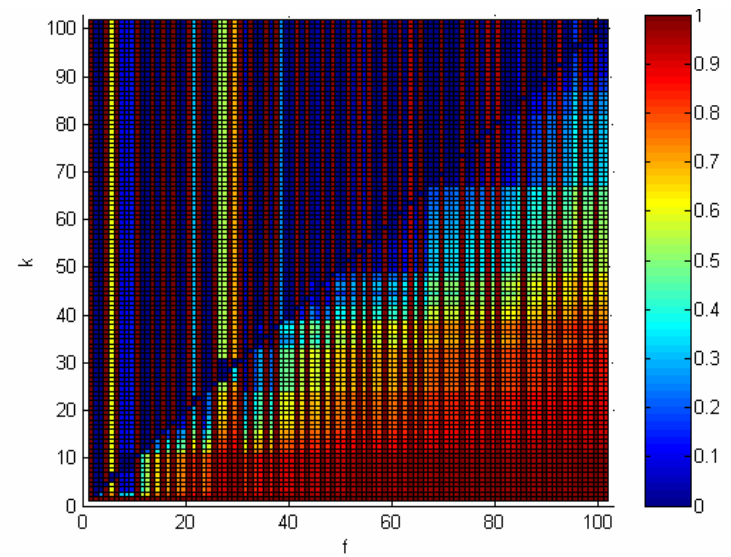

Fig. 4. Graphical visualization of the DFV matrix for case B

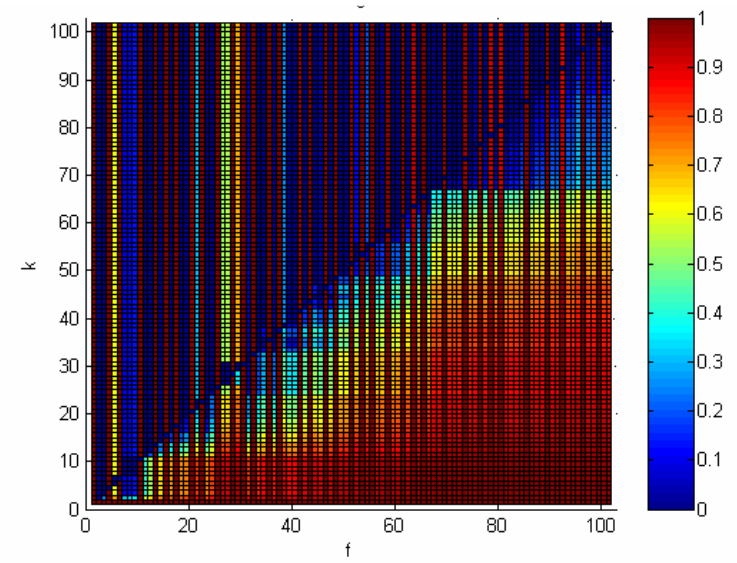

Fig. 5. Graphical visualization of the DFV matrix for case C

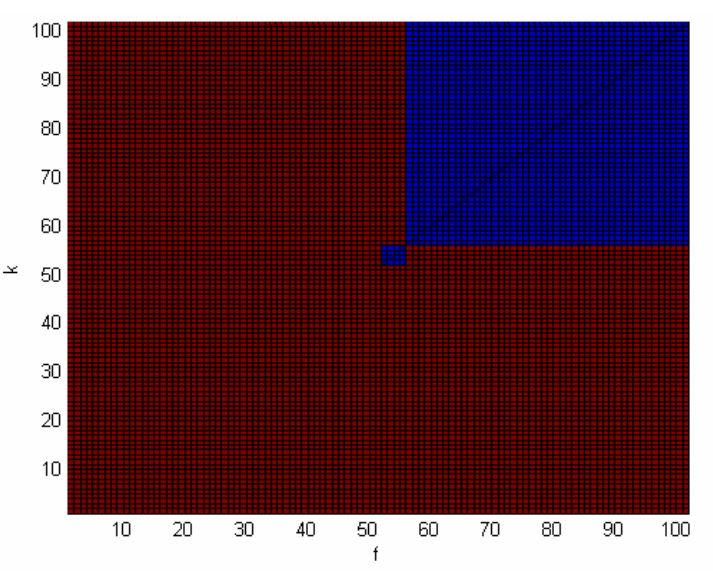

Fig. 6. Graphical visualization of the $\Delta X$ matrix 


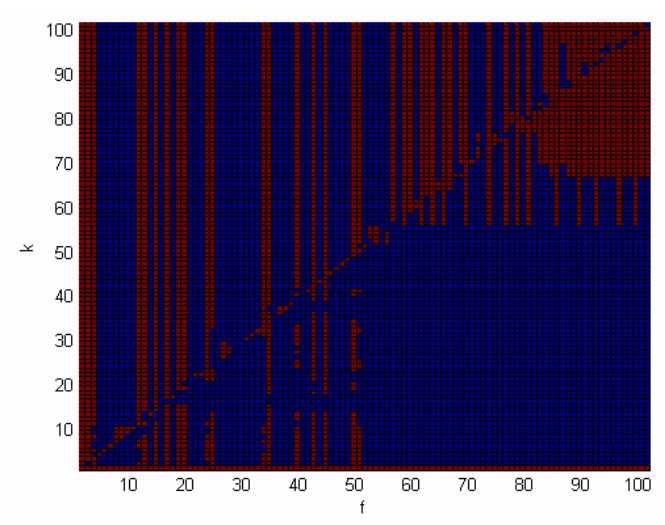

Fig. 7. Graphical visualization of the $\Delta V$ matrix

We firstly separate the nodes where the short circuit is increased (blue cells on the diagonal of the matrix in Fig.6) due to the changes in the network structure from the nodes where the short circuit power is not increased (red cells on the diagonal of the matrix in Fig.6).

i) Nodes in which the short circuit power is increased:

if the off-diagonal terms of the short circuit reactance are increased (red cells in Fig.6), the during fault voltages decrease (blue cell in Fig.7);

if the off-diagonal terms of the short circuit reactance are decreased (blue cells in Fig.6), then the terms $\frac{X^{\text {new }}(f, k)}{X(f, k)}$ have to be compared with $\frac{S_{S C}^{k}}{S_{S C}^{k^{n e w}}}$ in accord to the relation (8). With the help of Table I, we can have two sub-cases:

$\circ$ the during fault voltage after the structure variation, $V^{\text {new }}(f, k), \quad$ is less than the corresponding before the structure variation, $V(f, k)$, if $\frac{X^{\text {new }}(f, k)}{X(f, k)} \leq \frac{S_{S C}^{k}}{S_{S C}^{k}}$. In such a case the color of the the $\Delta \mathrm{V}$ matrix cells is blue.

$\circ$ the during fault voltage after the structure variation, $V^{\text {new }}(f, k)$, is greater than the corresponding before the structure variation, $V(f, k)$, if $\frac{X^{\text {new }}(f, k)}{X(f, k)} \geq \frac{S_{S C}^{k}}{S_{S C}^{k}}$.

In such a case the color of the the $\Delta \mathrm{V}$ matrix cells is red.

ii) Nodes in which the short circuit power is not increased.

if the off-diagonal terms of the short circuit reactance are decreased (blue cells in Fig.6), the during fault voltages increase (red cell not present in Fig.7);

if the off-diagonal terms of the short circuit reactance are increased (blue cells in Fig.6), then the terms $\frac{X^{\text {new }}(f, k)}{X(f, k)}$ have to be compared with $\frac{S_{S C}^{k}}{S_{S C}^{k}}$ in accord to the relation (8). With the help of Table I, we can have two sub-cases:

o the during fault voltage after the structure variation, $V^{\text {new }}(f, k)$, is less than the corresponding before the structure variation, $V(f, k)$, if $\frac{X^{\text {new }}(f, k)}{X(f, k)} \leq \frac{S_{S C}^{k}}{S_{S C}^{k}}$. In such a case the color of the the $\Delta V$ matrix cells is blue.

$\circ$ the during fault voltage after the structure variation, $V^{\text {new }}(f, k)$, is greater than the corresponding before the structure variation, $V(f, k)$, if $\frac{X^{\text {new }}(f, k)}{X(f, k)} \geq \frac{S_{S C}^{k}}{S_{S C}^{k}}$. In such a case the color of the the $\Delta V$ matrix cells is red.

The bicolor representation adopted in Fig.6 and Fig.7 can introduce some drawbacks mainly linked to numerical problems. For example, observing Fig.6 and Fig.7, it can be argued that a local change in the network (substitution of some overhead lines with cable lines) causes a huge propagation effect in terms of during fault voltage variation.

In reality, the variation of the elements of the matrices defined in (6) and (7) can be only numerical, but not really significant. This effect can be overcome

Table I: Analysis of the variations caused by change in the network and effect on the graphical visualization of (6) and (7).

\begin{tabular}{|c|c|c|c|c|c|}
\hline$\frac{X^{n e w}(k, f)}{X(k, f)}$ & Color of $\Delta X(k, f)$ cell & Increase of $S_{s c}^{f}$ & Color of $\Delta X(f, f)$ & $V_{k_{k}}^{\text {new }}-V_{k}$ & Color of $\Delta V_{\mu}$ \\
\hline$>1$ & Red & Yes & Blue & $<0$ & Blue \\
\hline \multirow{2}{*}{$>1$} & \multirow{2}{*}{ Red } & \multirow{2}{*}{ No } & \multirow{2}{*}{ Red } & $<0$ if $\frac{X^{\text {and }}(f, k)}{X(f, k)}>\frac{S_{s c}^{f}}{S_{s c}^{f(n e v e r}}$ & Blue \\
\hline & & & & 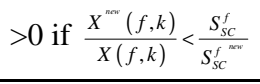 & Red \\
\hline \multirow{2}{*}{$<1$} & \multirow{2}{*}{ Blue } & \multirow{2}{*}{ Yes } & \multirow{2}{*}{ Blue } & $<0$ if $\frac{X^{\operatorname{loc}(f,}(f, k)}{X(f, k)}<\frac{S_{s c}^{f}}{S_{s c}^{f(x)}}$ & Blue \\
\hline & & & & $>0$ if $\frac{X^{\operatorname{now}(f)}(f, k)}{X(f, k)}>\frac{S_{S C}^{f}}{S_{S C}^{f(n+e n}}$ & Red \\
\hline$<1$ & Blue & No & Red & $>0$ & Red \\
\hline
\end{tabular}


introducing a proper threshold that brings to zero the values slightly different from zero. The results of this operation are shown in Fig.8 and Fig.9 for the matrix $\Delta X$ and $\Delta V$, respectively. The green color corresponds to zero values; the blue and red color have the same preceding means

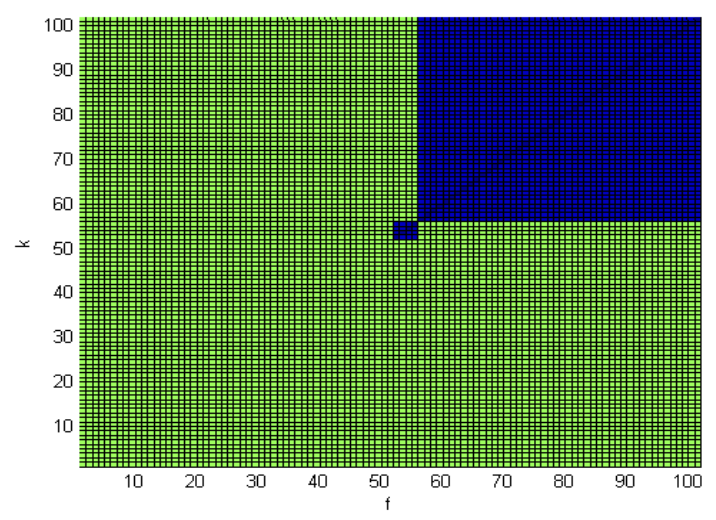

Fig. 8. Graphic visualization of $\Delta X$ matrix with a threshold

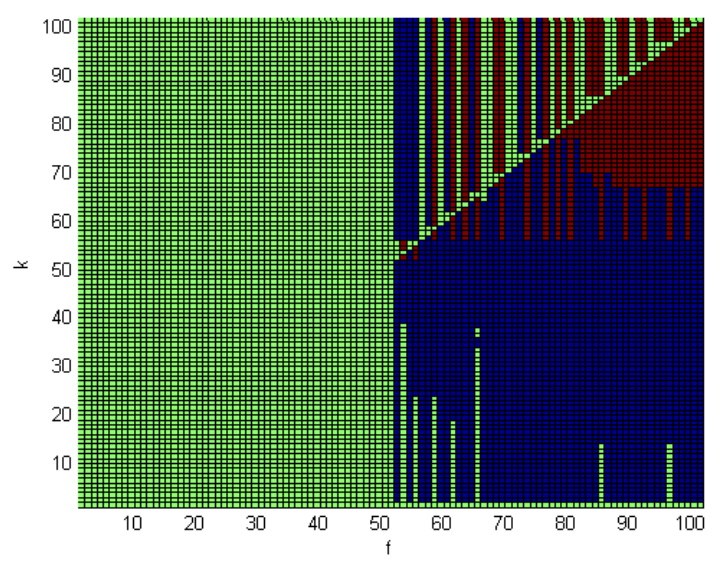

Fig. 9. Graphical visualization of $\Delta V$ matrix with a threshold

The results in Figures 8 and 9 confirm the analytical expressions in relationships (4) and (5) in a clearer manner.

Practically, when a fault originates in the busses from 1 to 51 , there is no variation on the during fault voltages (Fig.9); these are the nodes where the short circuit power has not changed (Fig.8). When a fault originates in a bus where the short circuit is increased (from 52 to 102) we can have different situations on the during fault voltages in dependence of the variations in the terms of diagonal of the matrix $X$. All the cases confirm that if the increase of the short circuit power and the variation of elements $(f, k)$ of the matrix $X$ are such that the relationship $X(f, k) \leq X^{\text {new }}(f, k) \frac{S_{S C}^{k} \text { new }}{S_{S C}^{k}} \quad$ is valid, then the performance in the other nodes in terms of voltage dips amplitude is worse, that is: $V^{\text {new }}(f, k) \leq V(f, k)$.

\section{Conclusion}

In this paper the concept of robustness against to the voltage dips has been applied. A graphical color schemes of the impedance variation matrices and of the during fault voltages variation matrices are presented as efficient tools to precisely foresee improvements and/or degradations of the electrical power system robustness when structure modifications are implemented.

The analysis of the specific properties of the variation of the Short Circuit reactance and the During Fault Voltage matrices allows better ascertaing the linkage between the short circuit power of each node and the electrical power system performance.

The numerical results of the robustness analysis on real electrical power systems confirm that the analyrical relationships are valid.

\section{References}

[1] M.H.J. Bollen, "Understanding power quality problems: voltage sags and interruptions", New York, IEEE Press, 1999.

[2] P. Verde, R. Chiumeo, I. Mastandrea, F. Tarsia, Definizione di procedure e metodologie per il monitoraggio della "robustezza" e dell'adeguatezza del sistema di potenza, CESI-Ricerca di sistema, Report A5-053071, www.ricercadisistema.it/documenti (in Italian), December 2005.

[3] G. Carpinelli, P. Caramia, C. Di Perna, P. Varilone, P. Verde, "Complete Matrix Formulation of fault-position method for voltage-dip characterization", IET Generation, Transmission \& Distribution, Volume 1, Issue 1, January 2007, pp. $56-64$.

[4] P. Caramia, G. Carpinelli, R. Chiumeo, C. Di Perna, I. Mastandrea, F. Tarsia, P. Varilone , P. Verde, On Robustness of Distribution Systems against Voltage Dips due to Faults, Proceedings of Speedam 2006, Taormina (IT), May 2006, pp. 1310 - 1315.

[5] P. Caramia, G. Carpinelli, C. Di Perna, P. Varilone, "Methods for Assessing the Robustness of Electrical Power Systems against Voltage Dips", IEEE Transactions on Power Delivery, Vol. 24, Issue 1, January 2009. 